\title{
Recent Charm Physics Results from CLEO
}

\author{
D.H. Fujino
}

This paper was prepared for submittal to the Sixth Conference on the Intersection of Particle and Nuclear Physics Big Sky, MT

May 22-June 2, 1997

July 1997

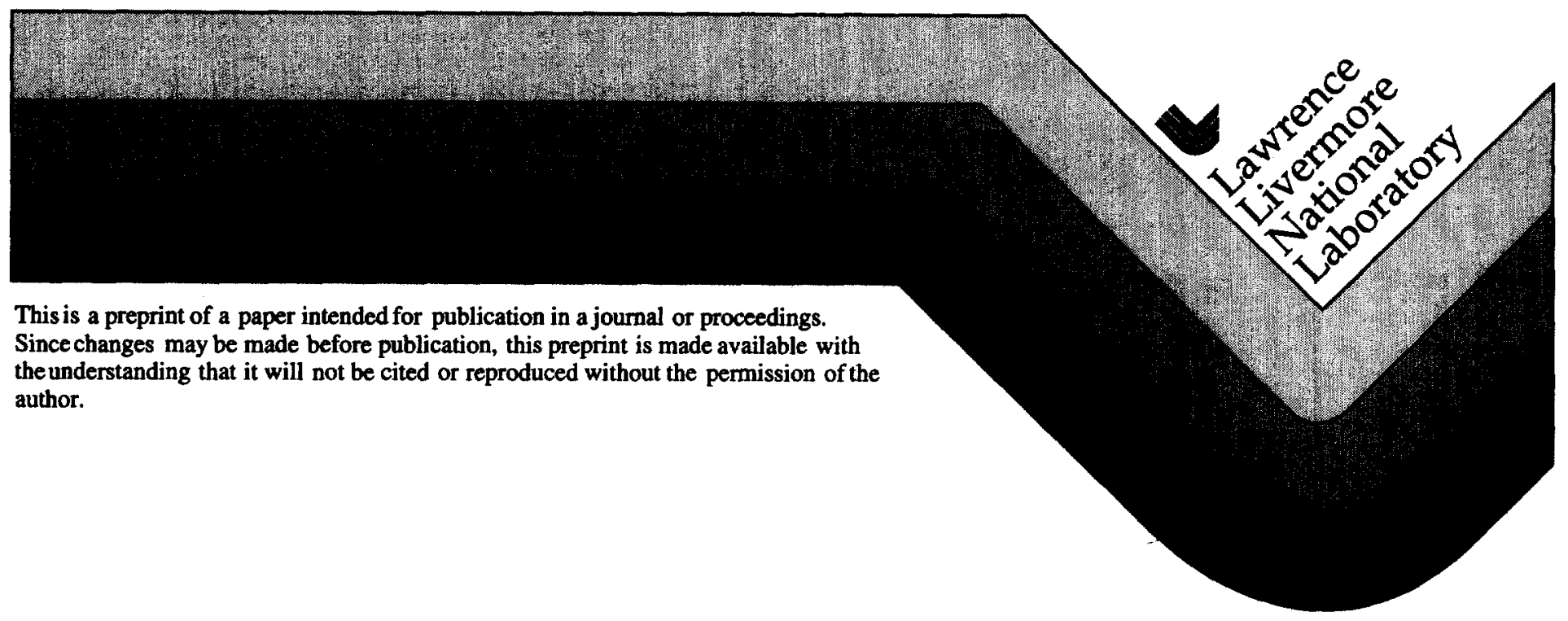


This document was prepared as an account of work sponsored by an agency of the United States Government. Neither the United States Government nor the University of California nor any of their employees, makes any warranty, express or implied, or assumes any legal liability or responsibility for the accuracy, completeness, or usefulness of any information, apparatus, product, or process disclosed, or represents that its use would not infringe privately owned rights. Reference herein to any specific commercial product, process, or service by trade name, trademark, manufacturer, or otherwise, does not necessarily constitute or imply its endorsement, recommendation, or favoring by the United States Government or the University of California. The views and opinions of authors expressed herein do not necessarily state or reflect those of the United States Government or the University of California, and shall not be used for advertising or product endorsement purposes. 


\title{
Recent Charm Physics Results from CLEO
}

\author{
Don H. Fujino ${ }^{1}$ \\ Lawrence Livermore National Laboratory \\ Livermore, $C A 94550$ \\ Representing the CLEO Collaboration
}

\begin{abstract}
In this talk I present recent charm physics results from the CLEO experiment. Final state interactions and $\mathrm{W}$-annihilation effects in charmed mesons decays are discussed. These include an isospin analysis of $D \rightarrow K \bar{K}$, observation of the candidate W-annihilation decay $D_{s}^{+} \rightarrow \omega \pi^{+}$, and evidence of nonfactorizable effects in $D_{s}^{+} \rightarrow \eta \pi^{+}, \eta^{\prime} \pi^{+}, \eta \rho^{+}$, and $\eta^{\prime} \rho^{+}$decays. Presented next are CLEO's observations of the spin $\frac{3}{2}^{+}$excited charmed baryons $\Sigma_{c}^{*++}$ and $\Sigma_{c}^{* 0}$, and the excited charmed-strange baryons $\Xi_{c}^{*+}$ and $\Xi_{c}^{* 0}$. I conclude with future prospects in charm physics with CLEO's new silicon detector.
\end{abstract}

\section{INTRODUCTION}

The external and internal spectator diagrams do an excellent job of explaining most of the features in charmed meson decays. For example, the lifetime hierarchy, i.e. the fact that the $D^{+}$meson lifetime is a factor of $2-2.5$ times longer than the $D^{0}$ or $D_{s}^{+}$lifetime can be attributed to the destructive interference between the external and internal diagrams for $D^{+}$decays. The $D^{+}$ decays $(c \bar{d} \rightarrow s u \bar{d} \bar{d})$ have two $\bar{d}$ quarks in the final state and so decays such as $D^{+} \rightarrow \bar{K}^{0} \pi^{+}$can occur through both the external and internal spectator diagrams. There is no interference in $D^{0}$ or $D_{s}^{+}$decays since the anti-quarks in the final state are not identical for $D^{0}$ decays $(c \bar{u} \rightarrow s u \bar{d} \bar{u})$ and $D_{s}^{+}$decays $(c \bar{s} \rightarrow s u \bar{d} \bar{s})$.

However, the charm quark is still light enough that non-factorizable effects can have non-negligible contributions when compared with the simple spectator decays in many exclusive decays of $D$ mesons. Two effects I will explore with the recent CLEO results are final state interactions (FSI) and $\mathrm{W}$-exchange and $\mathrm{W}$-annihilation decays.

1) This work performed under the auspices of the US Department of Energy by the Lawrence Livermore National Laboratory under Contract W-7405ENG-48. 


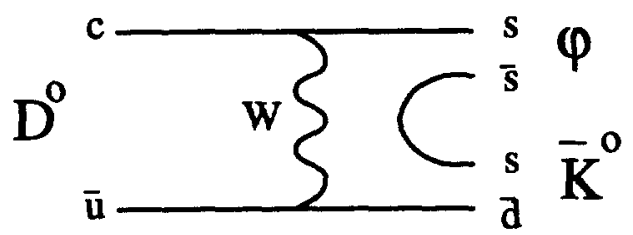

(a)

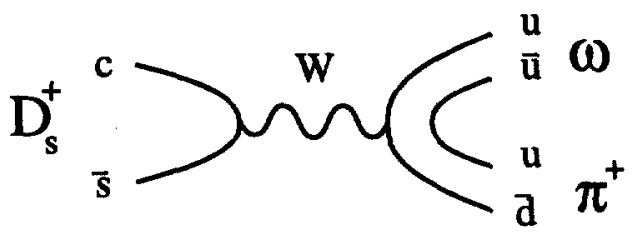

(b)

FIGURE 1. (a) W-exchange decay for $D^{0} \rightarrow \phi \bar{K}^{0}$ and (b) W-annihilation decay for $D_{s}^{+} \rightarrow \omega \pi^{+}$.

Final state interactions occur when the outgoing mesons get rescattered through intermediate resonances that lie near the $D$ mass. For example, the exiting kaon and pion in the $D^{0} \rightarrow K^{-} \pi^{+}$decay can get rescattered through a highly excited $\bar{K}^{0}$ state and emerge as the final state $\bar{K}^{0} \pi^{0}$. This can enhance color-suppressed decays, since FSI feeds into these channels from the colorallowed decays. For the $D \rightarrow K \pi$ system, $\mathcal{B}\left(D^{0} \rightarrow \bar{K}^{0} \pi^{0}\right) / \mathcal{B}\left(D^{0} \rightarrow K^{-} \pi^{+}\right)=$ $0.62 \pm 0.13$, which is much larger than the naive expectation of $1 / 9$ from color matching for quarks.

The above example is considered elastic FSI because the isospin and spin content of the initial and rescattered mesons do not change. On the other hand, inelastic FSI change the isospin and spin structure of the outgoing mesons. Some examples of inelastic FSI include $K \rho \leftrightarrow K^{*} \pi, K K \leftrightarrow \pi \pi$, and $K^{*} K^{*} \leftrightarrow K K$ rescattering.

W-exchange and W-annihilations diagrams for $D$ decays such as $D^{0} \rightarrow$ $\phi \bar{K}^{0}$ and $D_{s}^{+} \rightarrow \omega \pi^{+}$are shown in Figure 1. These diagrams are helicity suppressed compared to the spectator diagrams, and the ratio of decay rates is $\Gamma_{\text {annihilation }} / \Gamma_{\text {spectator }} \sim M_{q}^{2} / M_{D}^{2}$, where $M_{q}$ is the light quark mass. The only clear evidence of $\mathrm{W}$-annihilation decays is in the purely leptonic $D_{s}^{+}$ decays [1]. The $D_{s}^{+} \rightarrow \mu \nu$ decay has been observed by WA75, CLEO, BES, and most recently by E653 with a branching fraction of $\sim 0.5 \%$. The L3 Collaboration [2] has recently observed $D_{s}^{+} \rightarrow \tau \nu$ with a branching fraction of $(7.4 \pm 3.6) \%$.

\section{CHARM PHYSICS WITH CLEO}

The CLEO Collaboration consists of over 200 physicists from 24 universities. The data were selected from hadronic events collected by the CLEO II detector at the Cornell Electron Storage Ring (CESR). The CLEO II detector [3] is a large solenoidal detector with 67 tracking layers and a CsI electromagnetic calorimeter that provides efficient $\pi^{0}$ reconstruction. Charged kaon and pions are identified using specific ionization $(d E / d x)$ and, when available, time-offlight (TOF) information. The $d E / d x$ and TOF information provide good 
$K / \pi$ separation up to 0.7 and $1.1 \mathrm{GeV} / \mathrm{c}$, respectively. For high momentum particles the $d E / d x$ relativistic rise provides greater than $2 \sigma K / \pi$ separation, which is essential for separating rare $B$ processes such as $B^{0} \rightarrow \pi^{+} \pi^{-}$from $B^{0} \rightarrow K^{+} \pi^{-}$.

The data used in most of the analyses presented in this talk consist of an integrated luminosity of $4.8 \mathrm{fb}^{-1}$ taken at and just below the $\Upsilon(4 S)$ resonance, corresponding to $\sim 3 \times 10^{6} B \bar{B}$ events and $\sim 5 \times 10^{6} e^{+} e^{-} \rightarrow c \bar{c}$ events. In general, the charm physics analyses at CLEO use a common set of techniques to enhance the charmed hadron signal of interest. These include cuts on the $D$ momentum, event shape, and $D^{*}$ mass difference. The momentum spectrum for charmed hadrons from $e^{+} e^{-} \rightarrow c \bar{c}$ events is quite hard, so requiring the scaled $D$ momentum to satisfy $x_{p}=p_{D} / \sqrt{E_{\text {beam }}^{2}-M_{D}^{2}}>0.5$ removes a lot of the combinatoric background from low momentum tracks. Charmed hadrons from $B$ decays will have a momenta $x_{p}<0.5$, and as a side-effect get eliminated. The event shape can be used to enhance charm events since $e^{+} e^{-} \rightarrow c \bar{c}$ events are jetty, whereas $B \bar{B}$ events decay isotropically because the $B$ mesons are produced nearly at rest. Finally, the $D^{*}$ mass trick is a powerful tool to enhance the $D$ signal. The $D^{0}, D^{+}$, and $D_{s}^{+}$mesons are required to come from $D^{*+} \rightarrow D^{0} \pi^{+}, D^{*+} \rightarrow D^{+} \pi^{0}$, and $D_{s}^{*+} \rightarrow D_{s}^{+} \gamma$ decays, respectively. The $D^{*} \rightarrow D \pi$ decay has limited phase space and the resolution of the mass difference, $\Delta M=M_{D \pi}-M_{D}$, is on average 10 times better than the invariant mass of the reconstructed $D$ meson. So for example, a $D^{*+} \rightarrow D^{0} \pi^{+}$tag reduces the $D^{0}$ signal by $4 \times$ but reduces the background by $20-40 \times$.

\section{ISOSPIN ANALYSIS OF $D \rightarrow K K$}

CLEO [4] has recently measured the branching fraction to the Cabibboallowed decay $D^{+} \rightarrow K_{s}^{0} \pi^{+}$and the Cabibbo-suppressed decay $D^{+} \rightarrow K_{s}^{0} K^{+}$. To extract the signals, we require a $D^{*+} \rightarrow D^{+} \pi^{0}$ tag and $x_{p}>0.55$. We observe $70 \pm 12$ events for $D^{+} \rightarrow K_{s}^{0} K^{+}$and $473 \pm 26$ events for $D^{+} \rightarrow K_{s}^{0} \pi^{+}$ (shown in Figure 2.) The broad peak on the right of the $D^{+} \rightarrow K_{s}^{0} K^{+}$signal are $D^{+} \rightarrow K_{s}^{0} \pi^{+}$events where the pion is mis-identified as a kaon, and the excess on the left is due to feed-down from $D \rightarrow K_{s}^{0} \pi \pi$ events.

The branching fractions are computed to be $\mathcal{B}\left(D^{+} \rightarrow \bar{K}^{0} K^{+}\right)=(0.70 \pm$ $0.12 \pm 0.07 \pm 0.05) \%$ and $\mathcal{B}\left(D^{+} \rightarrow \bar{K}^{0} \pi^{+}\right)=(3.17 \pm 0.21 \pm 0.19 \pm 0.21 \pm$ $0.32) \%$, where the second error is systematic, the third is due to uncertainty in the normalization branching fraction, and the fourth error is due to possible interference from doubly Cabibbo suppressed decays (DCSD). The ratio of Cabibbo suppressed to allowed modes is $3.6 \sigma$ higher than $\tan ^{2} \theta_{C} \approx 0.05$. This is due to the destructive interference occurring in the $D^{+} \rightarrow \bar{K}^{0} \pi^{+}$decay mode.

Using CLEO's [1] previous results on $D^{0} \rightarrow K^{+} K^{-}, K^{0} \bar{K}^{0}$, and $\bar{K}^{0} \pi^{0}$, we have measured the isospin amplitudes and relative phase shift for $D \rightarrow K \bar{K}$ 

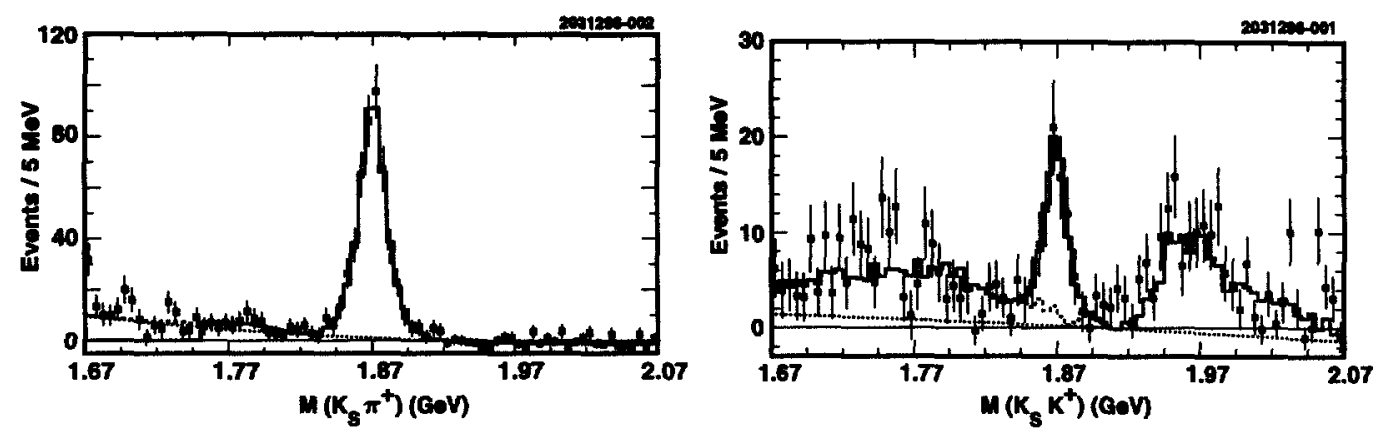

FIGURE 2. The invariant mass distribution for $K_{s}^{0} \pi^{+}$and $K_{s}^{0} K^{+}$combinations.

TABLE 1. Isospin analysis of $D \rightarrow K K, K \pi$, and $\pi \pi$.

\begin{tabular}{|c|c|c|c|}
\hline Decay Mode & Am & plitude Ratio & $\cos \delta$ \\
\hline$D \rightarrow K K$ & $\frac{A_{1}}{A_{0}}$ & $=0.61 \pm 0.11$ & $0.88 \pm 0.09$ \\
\hline$D \rightarrow K \pi$ & $\frac{A_{3 / 2}}{A_{1 / 2}}$ & $=0.27 \pm 0.03$ & $-0.12 \pm 0.22$ \\
\hline$D \rightarrow \pi \pi$ & $\left|\frac{A_{2}}{A_{0}}\right|$ & $=0.72 \pm 0.17$ & $0.14 \pm 0.16$ \\
\hline
\end{tabular}

for the first time, and improved the isospin analysis for the $D \rightarrow K \pi$ system. The three decay modes $D^{+} \rightarrow K^{+} K^{-}, D^{+} \rightarrow K^{0} \bar{K}^{0}$, and $D^{+} \rightarrow \bar{K}^{0} K^{+}$ can be described by two isospin amplitudes: $A_{0}$ and $A_{1}$, which correspond to the isospin 0 and 1 states for $K \bar{K}$. The decay rate amplitudes are linear combinations of the two isospin amplitudes.

$$
\begin{aligned}
A m p\left(D^{+} \rightarrow K^{+} K^{-}\right) & =\sqrt{1 / 2}\left(A_{1}+A_{0}\right) \\
A m p\left(D^{+} \rightarrow K^{0} \bar{K}^{0}\right) & =\sqrt{1 / 2}\left(A_{1}-A_{0}\right) \\
A m p\left(D^{+} \rightarrow \bar{K}^{0} K^{+}\right) & =\sqrt{2} A_{1}
\end{aligned}
$$

Similar isospin relations can be expressed for $D \rightarrow K \pi$ and $D \rightarrow \pi \pi$.

In the absence of FSI both $A_{0}$ and $A_{1}$ are real. When elastic FSI is turned on via resonant rescattering, the magnitude of the two isospin amplitudes do not change, but phase shifts can be introduced. This causes the $K^{+} K^{-}$and $K^{0} \bar{K}^{0}$ amplitudes to mix while the summed rate $\Gamma\left(D^{+} \rightarrow K^{+} K^{-}\right)+\Gamma\left(D^{+} \rightarrow K^{0} \bar{K}^{0}\right)$ stays unchanged. The $D^{+} \rightarrow \bar{K}^{0} K^{+}$amplitude remains invariant. Table 1 shows the isospin amplitude ratio and the relative phase shift $(\delta)$. The $\Delta I=$ $1 / 2$ rule in $K \rightarrow \pi \pi$ decays, which suppresses the $K^{+} \rightarrow \pi^{+} \pi^{0}$ decay and implies $\left|A_{2} / A_{0}\right| \approx 0.05$, does not hold for $D \rightarrow \pi \pi$ decays which has a large $A_{2}$ amplitude. The striking difference in behavior is not well understood.

In the $D \rightarrow K \pi$ and $\pi \pi$ systems, the color suppressed modes are apprecia- 


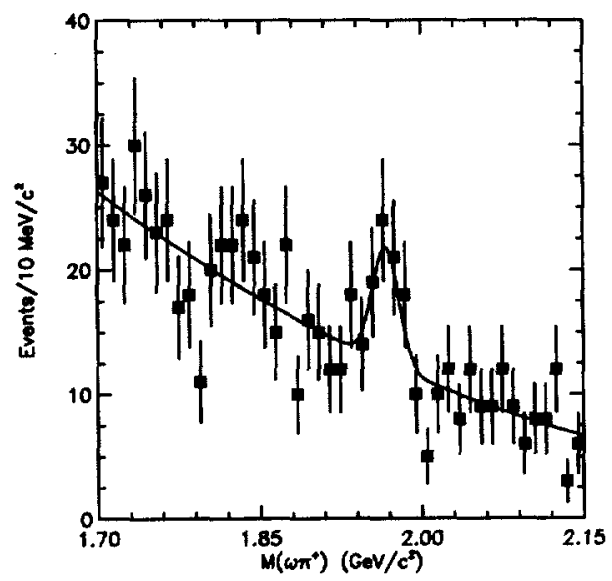

FIGURE 3. The invariant mass distribution for $\omega \pi^{+}$combinations from tagged $D_{B}^{*+}$ decays.

ble because $\delta \approx 90^{\circ}$. The ratio of color suppressed to color allowed branching fractions are large: $\mathcal{B}\left(D^{0} \rightarrow \bar{K}^{0} \pi^{0}\right) / \mathcal{B}\left(D^{0} \rightarrow K^{-} \pi^{+}\right)=0.62 \pm 0.13$ and $\mathcal{B}\left(D^{0} \rightarrow \pi^{0} \pi^{0}\right) / \mathcal{B}\left(D^{0} \rightarrow \pi^{+} \pi^{-}\right)=0.63 \pm 0.18$. Turning off elastic FSI by setting $\delta \rightarrow 0$ would decrease the ratios significantly to 0.14 and 0.00 , respectively.

On the other hand, the large ratio $\mathcal{B}\left(D^{0} \rightarrow K^{0} \bar{K}^{0}\right) / \mathcal{B}\left(D^{0} \rightarrow K^{+} K^{-}\right)=$ $0.12 \pm 0.03$ is unexpected. Because the $D^{0} \rightarrow K^{0} \bar{K}^{0}$ decay can only occur at tree-level via $\mathrm{W}$-exchange, assuming this rate is negligible we would expect $\left|A_{1} / A_{0}\right|=1$ and the $D^{0} \rightarrow K^{0} \bar{K}^{0}$ rate to be non-zero solely through FSI. But $\delta$ is consistent with being zero, so elastic FSI alone cannot be the explanation. Moreover, $\left|A_{1} / A_{0}\right|$ is $3.5 \sigma$ from unity. So either W-exchange contributions are large or "inelastic" FSI, which can change both the magnitude and phase of $A_{0}$ and $A_{1}$, are appreciable.

\section{OBSERVATION OF $D_{S}^{+} \rightarrow \omega \pi^{+}$}

$D_{s}^{+}$decays into final states with no strangeness are strong candidates for W-annihilation decays, since $D_{s}^{+}$spectator decays will always have an $\bar{s}$ quark in the final state. The $D_{s}^{+} \rightarrow \omega \pi^{+}$decay was expected to be a smoking gun for W-annihilation (see Figure 1) since the decay has no FSI contributions; the quantum numbers of the $\omega \pi^{+}$system $\left(J^{P}=0^{-}\right.$and $\left.I^{G}=1^{+}\right)$do not correspond to any known resonances. This is not true for $D_{s}^{+} \rightarrow \pi \pi$ or $\rho \pi$ which are expected to have significant FSI.

However, this simple model is flawed. The $u \bar{d}$ quark pair from Wannihilation cannot hadronize into $\omega \pi^{+}$due to G-parity conservation, which would require a second-class axial current. If, however, three gluons are connected to the initial state $c \bar{s}$ quark line, the modified W-annihilation process can occur with $W^{+} \rightarrow u \bar{d} \rightarrow \pi^{+}$and $g g g \rightarrow \omega$. Since the gluons also can carry 
spin, this process is no longer helicity suppressed. To further complicate the picture, although resonant FSI are not possible, Kamal et al. [5] and Buccella et al. [6] have suggested that non-resonant FSI can feed into $D_{s}^{+} \rightarrow \omega \pi^{+}$in the range $0.3-3 \%$.

CLEO [7] has searched for the decay $D_{s}^{+} \rightarrow \omega \pi^{+}$with $\omega \rightarrow \pi^{+} \pi^{-} \pi^{0}$. Only $\omega \rightarrow \pi^{+} \pi^{-} \pi^{0}$ combinations in the central Dalitz region were selected, and $\omega \pi^{+}$candidates were required to come from $D_{s}^{*+}$ decays. We observed $36 \pm 10$ $D_{s}^{+} \rightarrow \omega \pi^{+}$events, shown in Figure 3. To verify there is no contribution from other $D_{s}^{+} \rightarrow 4 \pi$ decays, the $\omega$ mass cut was loosened and the $4 \pi$ invariant mass was required to be in the $D_{s}^{+}$signal region. A fit to the $3 \pi$ invariant mass after a $D_{s}^{+}$sideband subtraction yielded a comparable $\omega$ signal of $32 \pm 12$ events. The $\omega \pi^{+}$combinations that failed the $D_{s}^{*+}$ tag still contain an excess of $133 \pm 57$ events at the $D_{s}^{+}$mass. The tagged and untagged results were combined and the branching fraction was normalized to the $D_{s}^{+} \rightarrow \eta \pi^{+}$decay to obtain $\mathcal{B}\left(D_{s}^{+} \rightarrow \omega \pi^{+}\right) / \mathcal{B}\left(D_{s}^{+} \rightarrow \eta \pi^{+}\right)=0.16 \pm 0.04 \pm 0.03$. This is the first measurement of $D_{s}^{+} \rightarrow \omega \pi^{+}$. The decay cannot occur from a simple spectator diagram, but must be due to FSI and/or W-annihilation.

\section{MEASUREMENT OF $D_{S}^{+} \rightarrow \eta \pi^{+}, \eta^{\prime} \pi^{+}, \eta \rho^{+}$, AND $\eta^{\prime} \rho^{+}$}

The decays $D_{s}^{+} \rightarrow \eta \pi^{+}, \eta^{\prime} \pi^{+}, \eta \rho^{+}$, and $\eta^{\prime} \rho^{+}$comprise $25-30 \%$ of the total $D_{s}^{+}$branching fraction. An earlier CLEO analysis showed a $2 \sigma$ departure from the factorization hypothesis that relates the two-body hadronic decay $D_{s}^{+} \rightarrow \eta^{\prime} \rho^{+}$to the semileptonic decay $D_{s}^{+} \rightarrow \eta^{\prime} \ell \nu$ :

$$
\Gamma\left(D_{s}^{+} \rightarrow \eta^{\prime} \rho^{+}\right)=\left.6 \pi^{2} a_{1}^{2} f_{\rho}^{2}\left|V_{u d}\right|^{2} \frac{d \Gamma}{d q^{2}}\left(D_{s}^{+} \rightarrow \eta^{\prime} \ell \nu\right)\right|_{q^{2}=m_{\rho}^{2}}
$$

CLEO measured $\Gamma\left(D_{s}^{+} \rightarrow \eta^{\prime} \rho^{+}\right) / \Gamma\left(D_{s}^{+} \rightarrow \eta^{\prime} \ell \nu\right)=14.8 \pm 5.8$ and $\Gamma\left(D_{s}^{+} \rightarrow\right.$ $\left.\eta \rho^{+}\right) / \Gamma\left(D_{s}^{+} \rightarrow \eta \ell \nu\right)=4.3 \pm 1.1$, whereas theory predicts both ratios to be $2.9[8]$.

With the full CLEO II dataset we have reexamined these branching fractions and the factorization hypothesis. For the $D_{s}^{+} \rightarrow \eta \pi^{+}$and $\eta^{\prime} \pi^{+}$modes, the $\eta$ is reconstructed in the $\gamma \gamma$ and $\pi^{+} \pi^{-} \pi^{0}$ channels. To construct the $\eta \rho^{+}$ and $\eta^{\prime} \rho^{+}$invariant mass distributions, only the $\eta \rightarrow \gamma \gamma$ mode was used. Nonresonant $\eta^{(\prime)} \pi^{+} \pi^{0}$ contributions were reduced by requiring the $\pi^{+} \pi^{0}$ mass to be within $170 \mathrm{MeV} / \mathrm{c}^{2}$ of the $\rho^{+}$mass and the helicity angle to be $\left|\cos \theta_{\pi}\right|>0.45$ since the $\rho^{+}$must have zero helicity. There can still be non-negligible nonresonant feedthrough, so the $D_{s}^{+} \rightarrow \eta \rho^{+}$and $\eta^{\prime} \rho^{+}$branching fractions are extracted by relaxing the $\rho$ cuts and fitting the Dalitz plot. Shown in Table 2 are the measured branching fractions along with the theoretical predictions. The variety of theoretical predictions assume form factors for the two-body decays from the pole model and from semileptonic decays. The predictions are consistent with the $\eta \pi^{+}, \eta^{\prime} \pi^{+}$, and $\eta \rho^{+}$branching fractions but cannot explain 
TABLE 2. CLEO results and theoretical predictions for the $D_{s}^{+} \rightarrow \eta \pi^{+}, \eta^{\prime} \pi^{+}, \eta \rho^{+}$, and $\eta^{\prime} \rho^{+}$branching fractions.

\begin{tabular}{cccccc}
\hline$D_{s}^{+}$ & \multicolumn{5}{c}{$\Gamma / \Gamma\left(D_{a}^{+} \rightarrow \phi \pi^{+}\right)$} \\
\cline { 2 - 6 } Mode & CLEO & VKK $[9]$ & BSW [10] & BLP [6] & HK [11] \\
\hline$\eta \pi^{+}$ & $0.48 \pm 0.03 \pm 0.04$ & 0.9 & 1.0 & 0.3 & 0.6 \\
$\eta^{\prime} \pi^{+}$ & $1.03 \pm 0.06 \pm 0.07$ & 0.8 & 0.6 & 1.3 & 1.6 \\
$\eta \rho^{+}$ & $2.98 \pm 0.20 \pm 0.39$ & 2.0 & 2.0 & 1.8 & 2.9 \\
$\eta^{\prime} \rho^{+}$ & $2.78 \pm 0.28 \pm 0.30$ & 0.7 & 0.6 & 0.6 & 0.4 \\
\hline
\end{tabular}

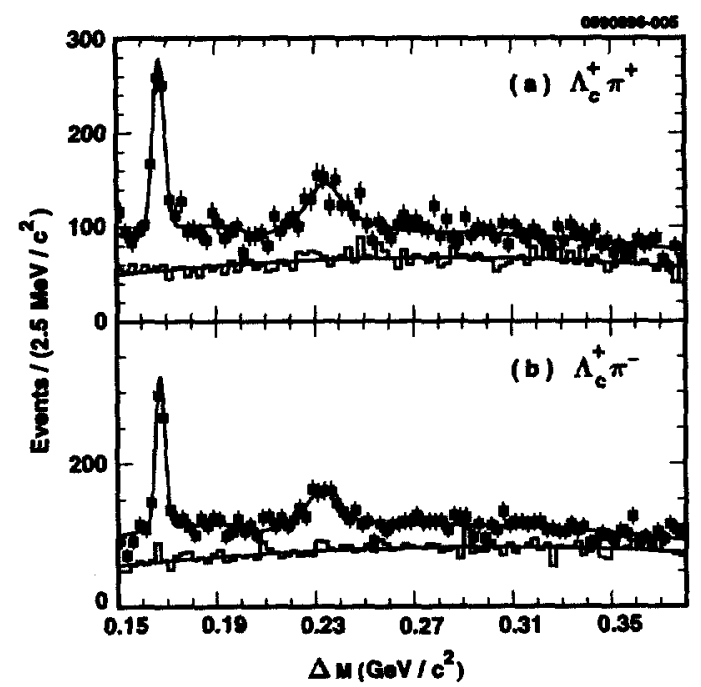

FIGURE 4. Mass difference spectra for (a) $\Lambda_{c}^{+} \pi^{+}$candidates and (b) $\Lambda_{c}^{+} \pi^{-}$candidates. The histogram shows the spectra for normalized sidebands of the $\Lambda_{c}^{+}$.

the large rate measured for $D_{s}^{+} \rightarrow \eta^{\prime} \rho^{+}$. Nor can FSI both resonant and non-resonant explain the discrepancy. In terms of the original factorization hypothesis, we now measure $\Gamma\left(D_{s}^{+} \rightarrow \eta^{\prime} \rho^{+}\right) / \Gamma\left(D_{s}^{+} \rightarrow \eta^{\prime} \ell \nu\right)=12.0 \pm 4.3$ and $\Gamma\left(D_{s}^{+} \rightarrow \eta \rho^{+}\right) / \Gamma\left(D_{s}^{+} \rightarrow \eta \ell \nu\right)=4.4 \pm 1.2$, compared with the theoretical prediction of 2.9. The uncertainty is primarily due to the uncertainty in the measured semileptonic decay rates. Ball et al. [12] has suggested that a modified $\mathrm{W}$-annihilation diagram may be enhancing this mode, where two gluons connected to the initial $c \tilde{s}$ quark line hadronize to the $\eta^{\prime}$. This is analogous to the $\mathrm{W}$-annihilation diagram for $D_{s}^{+} \rightarrow \omega \pi^{+}$.

\section{CHARMED BARYON SPECTROSCOPY}

The family of charmed baryons is quite diverse. There is the isosinglet $\Lambda_{c}^{+}$ and the isotriplet $\Sigma_{c}$, the charmed-strange baryons $\Xi_{c}$ and $\Xi_{c}^{\prime}$, and finally the 

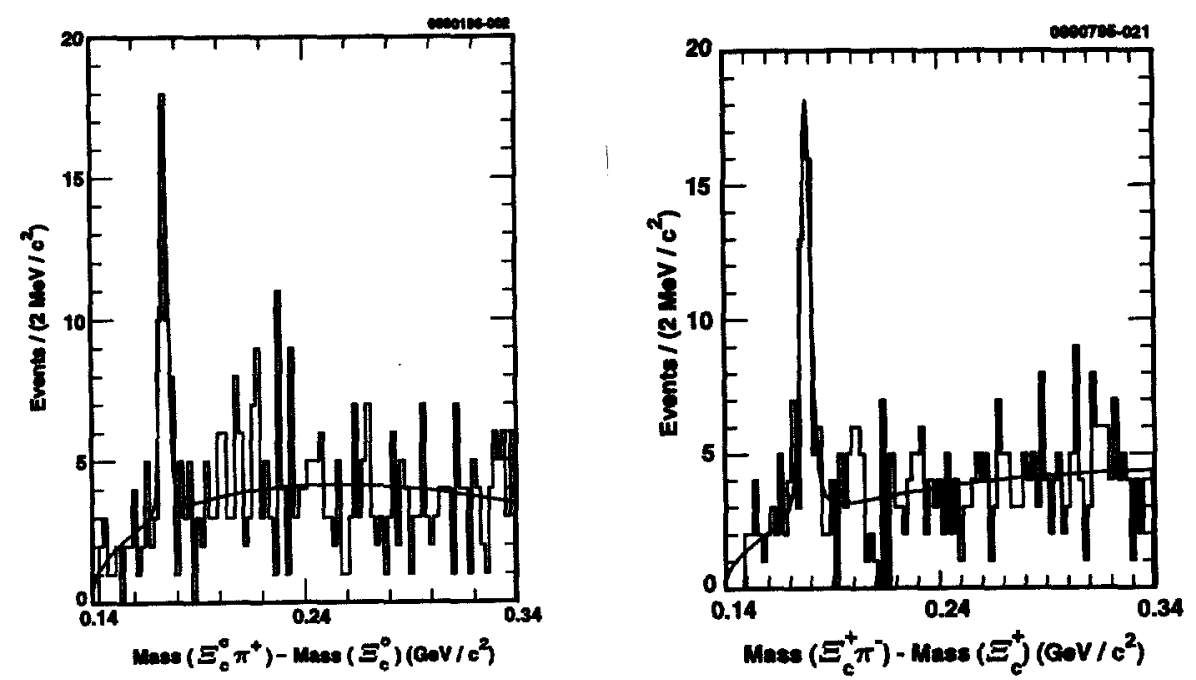

FIGURE 5. Mass difference spectra for (a) $\Xi_{c}^{0} \pi^{+}$candidates and (b) $\Xi_{c}^{+} \pi^{-}$candidates.

doubly-strange charmed baryon $\Omega_{c}$. Most of the ground state charmed baryons with $J^{P}=\frac{1}{2}^{\text {}}$ have been well measured. CLEO [13-15] has now observed many of the spin $\frac{3}{2}^{+}$excitations of charmed baryons. These include the $\Sigma_{c}^{*++}$ and $\Sigma_{c}^{* 0}$ which decay into $\Lambda_{c}^{+} \pi^{+}$and $\Lambda_{c}^{+} \pi^{-}$, respectively; and the charmed-strange baryons $\Xi_{c}^{*+}$ and $\Xi_{c}^{* 0}$ which are observed via the decay into $\Xi_{c}^{0} \pi^{+}$and $\Xi_{c}^{+} \pi^{-}$, respectively. The $\Sigma_{c}^{*++}$ and $\Sigma_{c}^{* 0}$ are part of an isospin triplet, but the $\Sigma_{c}^{*+}$ has yet to be seen, primarily because of the difficulty of reconstructing its decay to $\Lambda_{c}^{+} \pi^{0}$.

The search for these spin excitations is similar to reconstructing $D^{*}$ mesons. We accumulate large samples of the ground state charmed baryons, add a charged pion, apply an $x_{p}>0.5 \mathrm{cut}$, and examine the mass difference spectrum. For the $\Sigma_{c}^{*++}$ and $\Sigma_{c}^{* 0}$ search, we start with $\sim 15000 \Lambda_{c}^{+}$candidates from 13 decay modes. These include $\Lambda_{c}^{+} \rightarrow p K^{-} \pi^{+}(\sim 8400$ evts $), p K_{s}^{0}(\sim 1000$ evts), $\Lambda \pi^{+}\left(\sim 1100\right.$ evts), and $\Lambda \pi^{+} \pi^{0}$ ( $\sim 900$ evts). The $\Lambda_{c}^{+} \pi^{+}$like-sign and $\Lambda_{c}^{+} \pi^{-}$unlike-sign mass difference distributions are shown in Figure 4. Results of the fits to the $\Sigma_{c}^{*}$ signals with a Breit-Wigner convoluted with the detector resolution are shown in Table 3 . The broad peaks at $\Delta M \approx 233 \mathrm{MeV} / \mathrm{c}^{2}$ are the signals for $\Sigma_{c}^{*++}$ and $\Sigma_{c}^{* 0}$, in very good agreement with theoretical predictions. Both the $\Lambda_{c}^{+} \pi^{+}$and $\Lambda_{c}^{+} \pi^{-}$have nearly identical mass splittings, as is expected for isospin partners. The narrow peak at $\Delta M \approx 167 \mathrm{MeV} / \mathrm{c}^{2}$ is the ground state $\Sigma_{c}$. The $\Sigma_{c}^{*}$ natural width is large because of the available phase space.

To search for the excited $\Xi_{c}^{*+}$ and $\Xi_{c}^{* 0}$ baryons, we collect a large sample of the ground state $\Xi_{c}^{+}$and $\Xi_{c}^{0}$ baryons. We use about $300 \Xi_{c}^{+}$candidates from the decays $\Xi_{c}^{+} \rightarrow \Xi^{-} \pi^{+} \pi^{+}, \Xi^{0} \pi^{+} \pi^{0}$, and $\Sigma^{+} K^{* 0}$; and about $300 \Xi_{c}^{0}$ candidates from the decays $\Xi_{c}^{0} \rightarrow \Xi^{-} \pi^{+}, \Omega^{-} K^{+}, \Xi^{-} \pi^{+} \pi^{0}$, and $\Xi^{0} \pi^{+} \pi^{-}$. Combining 
TABLE 3. Spin $\frac{3}{2}^{+}$excited charmed baryons: $\Sigma_{c}^{*}$ and $\Xi_{c}^{*}$.

\begin{tabular}{cccc}
\hline Decay Mode & Signal (evts) & $\Delta M\left(\mathrm{MeV} / \mathrm{c}^{2}\right)$ & $\Gamma\left(\mathrm{MeV} / \mathrm{c}^{2}\right)$ \\
\hline$\Sigma_{c}^{*++} \rightarrow \Lambda_{c}^{+} \pi^{+}$ & $677_{-93}^{+101}$ & $234.5 \pm 1.1 \pm 0.8$ & $17.9_{-3.2}^{+3.8} \pm 4.0$ \\
$\Sigma_{c}^{* 0} \rightarrow \Lambda_{c}^{+} \pi^{-}$ & $504_{-83}^{+93}$ & $232.6 \pm 1.0 \pm 0.8$ & $13.0_{-3.0}^{+3.7} \pm 4.0$ \\
$\Xi_{c}^{*+} \rightarrow \Xi_{c}^{0} \pi^{+}$ & $35 \pm 9$ & $174.3 \pm 0.5 \pm 1.0$ & $<3.9$ \\
$\Xi_{c}^{* 0} \rightarrow \Xi_{c}^{+} \pi^{-}$ & $55 \pm 12$ & $178.2 \pm 0.5 \pm 1.0$ & $<5.5$ \\
\hline
\end{tabular}

the $\Xi_{c}$ candidate with a charged pion and calculating the mass difference $\Delta M=M\left(\Xi_{c} \pi\right)-M\left(\Xi_{c}\right)$, we observe a narrow peak at $\Delta M=174.3 \pm 0.5 \pm 1.0$ for $\Xi_{c}^{0} \pi^{+}$and a narrow peak at $\Delta M=178.2 \pm 0.5 \pm 1.0$ for $\Xi_{c}^{+} \pi^{-}$combinations (see Figure 5 and Table 3). We identify these states as the $\Xi_{c}^{*+}$ and $\Xi_{c}^{* 0}$, respectively, because the theoretical mass predictions are consistent with our measurements and the two mass differences are nearly identical as is expected for isospin partners.

\section{FUTURE PROSPECTS IN CHARM PHYSICS}

In the Fall of 1995 the Silicon Vertex detector (SVX) was installed in the CLEO detector, and we have to date collected $\sim 3 \mathrm{fb}^{-1}$ of data, roughly $60 \%$ of the CLEO II dataset. The SVX is a three-layer, double-sided silicon strip detector that provides both $r \phi$ and $z$ measurements. This will give a big boost to the charm physics program at CLEO. For the first time we will have decay length information for charmed hadrons. Vertex contrained fits to three-prong $D^{+}$or two-prong $D^{0}$ decays will greatly reduce the combinatoric backgrounds. The $D^{*+} \rightarrow D^{0} \pi^{+}$mass difference resolution has improved three-fold due to the SVX performance, Kalman track fitting, and vertex contrained fitting. Finally, the CLEO drift chamber is now using a helium-based gas in place of Argon-Ethane, which reduces the multiple scattering and reduces the Lorentz angle to provide improved hit efficiency over the entire drift cell.

Below is a wish list of future charm physics analyses that will benefit strongly from the SVX and improved tracking capabilities.

- Measure the $D^{0}$ and $D^{+}$lifetimes with $1-2 \%$ precision

- Untangle the DCSD and $D^{0} \bar{D}^{0}$ mixing contributions to $D^{0} \rightarrow K^{+} \pi^{-}$ decays from the decay time information.

- Measure the natural width of $D^{*+}$ to a precision of $\sim 50 \mathrm{KeV}$

- Measure Cabibbo suppressed decays such as $D^{+} \rightarrow \omega \ell^{+} \nu$

- Analyze $D^{+} \rightarrow \bar{K}^{* 0} \ell^{+} \nu$ form factors 
The CLEO detector continues to evolve and will undergo a major upgrade in two years time when basically everything inside the CsI calorimeter will be replaced. The CESR peak luminosity is currently $4 \times 10^{32} \mathrm{~cm}^{-2} \mathrm{~s}^{-1}$; there is steady progress to increase the luminosity by a factor of 10 . This will necessitate new rare earth and superconducting quadrupole magnets in the final focus. CLEO will build a new four-layer silicon detector and drift chamber which uses a helium-based gas. Finally, to identify pions and kaons with $>4 \sigma$ separation over the entire momentum and $\cos \theta$ range a ring imaging Cerenkov detector will be constructed.

In summary, the recent CLEO results in charm physics have explored final state interactions and $\mathrm{W}$-annihilation processes in charmed meson decays. Isospin analyses of $D \rightarrow K \bar{K}, K \pi$, and $\pi \pi$ show significant FSI. The candidate W-annihilation decay $D_{s}^{+} \rightarrow \omega \pi^{+}$has been observed. The $D_{s}^{+} \rightarrow \eta^{\prime} \rho^{+}$decay rate cannot be explained by factorization and may have a decay mechanism similar to that of $D_{s}^{+} \rightarrow \omega \pi^{+}$. We have now observed many of the spin $\frac{3}{2}^{+}$ excited charmed baryons: $\Sigma_{c}^{*++}, \Sigma_{c}^{* 0}, \Xi_{c}^{*+}$, and $\Xi_{c}^{* 0}$. Discovery of the missing spectral states including the $\Xi_{c}^{\prime}$ and new orbitally excited charmed baryons are just around the corner. With luminosity and detector advancements, CLEO will continue to have a rich charm physics program in the years to come.

Acknowledgements: I would like to thank H. Yamamoto, J. Urheim, L. Gibbons, J. Bartelt, D. Kim, and M. Bishai for useful and stimulating discussions.

\section{REFERENCES}

1. Particle Data Group, R.M. Barnett et al., Phys. Rev. D 54, 1 (1996).

2. L3 Collaboration, preprint CERN-PPE/96-198.

3. Y. Kubota et al., Nucl. Inst. and Meth. A320, 66 (1992).

4. M. Bishai et al., Phys. Rev. Lett. 78, 3261 (1997).

5. A.N. Kamal, N. Sinha, and R. Sinha, Phys. Rev. D 39, 3503 (1989).

6. F. Buccella, M. Lusignoli, and A. Pugliese, Phys. Lett. B 379, 249 (1996).

7. R. Balest et al., preprint CLNS 97/1479, CLEO 97-8.

8. A.N. Kamal, Q.P. Xu, and A. Czarnecki, Phys. Rev. D 49, 1330 (1994).

9. R.C. Verma, A.N. Kamal, and M.P. Khanna, Z. Phys. C 65, 255 (1995).

10. M. Bauer, B. Stech, and M. Wirbel, Z. Phys. C 34, 102 (1987).

11. I. Hinchliffe and T.A. Kaeding, Phys. Rev. D 54, 914 (1996).

12. P. Ball, J.M. Frere, and M. Tytgat, Phys. Lett. B 365, 367 (1996).

13. G. Brandenburg et al., Phys. Rev. Lett. 78, 2304 (1997).

14. L. Gibbons et al., Phys. Rev. Lett. 77, 810 (1996).

15. P. Avery et al., Phys. Rev. Lett. 75, 4364 (1995). 


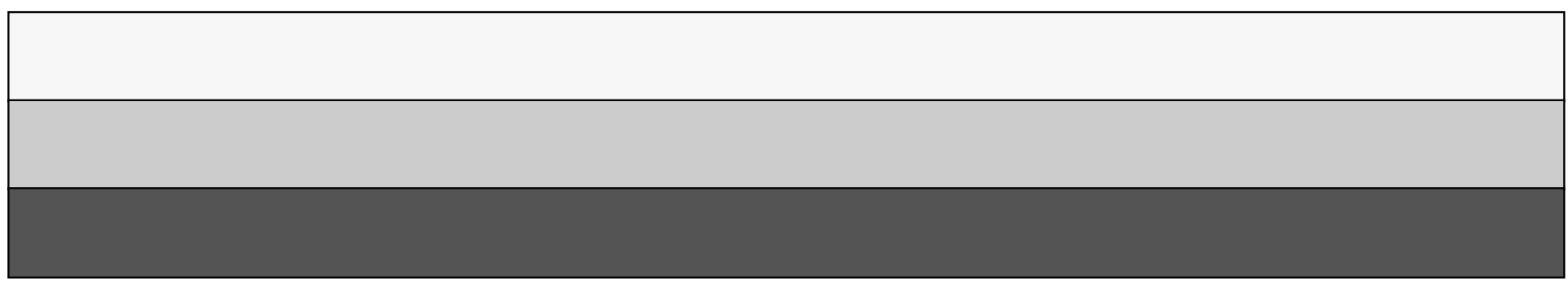

